\title{
Mechanical Analysis of Power Electromagnetic Contactors
}

\author{
Adrian T. Plesca* \\ Department of Power Engineering, Gheorghe Asachi Technical University of lasi, \\ Romania; matrix_total2000@yahoo.com
}

\begin{abstract}
This paper presents an analysis of the drive mechanism of an AC three-phase electromagnetic contactor. One of the keyfeature of the drive mechanism is the strength characteristic, which has been established from experimental tests. A good correlation between strength torque characteristic and the mechanical characteristics of the drive coil for different voltage supply (from 1.1 to 0.7 of rated voltage) has been observed. The recorded oscillograms of the time evolution of the trip of the movable armature, allow to obtain the connection time and the disconnection time of the contactor. Also, the drive coil has been supplied with variable voltage (from 1.1 to 0.7 of rated voltage). The connection and disconnection times are important parameters related to the contactor behaviour from mechanical point of view.
\end{abstract}

Keywords: Electromagnetic Contactor, Drive Mechanism, Trip Characteristic.

\section{Introduction}

Contactors with AC coils are among the most widely utilized electromechanical devices in industry. However, since this kind of equipment is usually very sensitive to disturbances in the utility voltages, they can be responsible for load misoperation and loss of production, [1-3]. An electronic device to protect $\mathrm{AC}$ contactors from power disturbances, such as voltage sags, is presented in [4]. The use of a square-wave series voltage compensator in the protection of control panels against voltage sags is discussed in [5]. Contactors are designed to disconnect the load or circuit they control when the main power supply is intentionally interrupted. The sensitivity of ac coil contactors to the applied voltage is thus a potentially weak link in industrial processes. An analysis of extensive laboratory testing carried out on ac coil contactors and switch-mode power supply of a PC system to voltage sags with variation in value of point on wave angle of voltage sags, is presented in [6]. Copper-tungsten contacts are frequently used in high power circuit breakers and contactors in air, oil, $\mathrm{SF}_{6}$, or vacuum. Arc interruption and anti-welding properties are important design parameters. In [7], the effects of tungsten particle size on reignition and weld resistance in air have been examined. Higher power density and longer electrical lifetime at the same time are the main technical targets in development of contactors. Therefore, a close matching of contact material and switching device as well as the understanding of the material-device-interactions is necessary to achieve those demands. The general influence of different material parameters based on $\mathrm{Ag} / \mathrm{SnO}_{2}$ in model switch tests and off-the-shelf contactors under various load conditions, is demonstrated in $[8,9]$. The fight for ecological solutions is lead in every technical field. Until 30 years ago, the challenge in the field of power switching apparatus was the interrupting currents and voltages increasing. Nowadays it is important to maintain the same technical performances but to use ecological constructive solutions. In low and medium voltage (up to $36 \mathrm{kV}$ ) the switching in vacuum is an ecological very

*Corresponding author:

Adrian T. Plesca (matrix_total2000@yahoo.com) 
good solution, but it raises technical and technological difficulties. For the low voltage contactors the replacement of the $\mathrm{Ag}-\mathrm{CdO}$ contact material with $\mathrm{Ag}-\mathrm{SnO}_{2}$ is the recommended ecological solution $[10,11]$. AC-powered contactors are extensively used in industry in applications such as automatic electrical devices, motor starters, and heaters. In [12], a practical model and simulation of the dynamic behaviour of ac-powered electromechanical contactors is presented. A dynamic simulator for analyzing various behaviours of electromagnetic contactors with AC solenoids, is presented in [13]. The simulator enables shorttime analysis of dynamic motions of contactors compared with conventional simulation methods such as finite element method. The study of current and voltage waveforms while controlling relays and low-voltage contactors using a LabVIEW environment, is described in $[14,15]$. A fast and effective method for detecting and isolating faults in multi-terminal medium voltage dc shipboard power distribution systems is presented in $[16,17]$. This method allows converters and contactors to use only local measurements when deciding whether or not to trip in order to isolate the faulted section. One of the trends in telecommunications power is towards small and compact power systems, [18]. The compact power system needs to be redefined and re-engineered because of the trend of electronics to move from the central office to the loop. To achieve this goal, the system concept has to be optimized, including all the range of contactors, $[19,20]$. In an induction-motor-based spindle drive for machine-tool applications, the wye/ delta switchover method remains popular for extending the constant-power range without sacrificing the torque capability at higher speeds, [21, 22]. A new generation of medium voltage vacuum circuit breakers can be used to replace sealed $\mathrm{SF}_{6}$ contactors which experience difficulty in disconnecting the loads safely after 15 years of service, [23].

This paper presents a study related to the drive mechanical system of a power AC three-phase contactor. The connecting and disconnection trip characteristics under various voltage supply values of the drive coil, have been investigated.

\section{Theoretical Aspects}

The kinematics' analysis of drive mechanism means to establish the kinematics' diagram, the energy sources and the position of the component parts, experimental validation and the motion diagrams. To analyze the mechanism behaviour during transient conditions means to establish the reduction of the masses, of inertia moments, of forces and their moments to a reference element considering the conservation of kinetic energy. Hence, the kinetic energy for a single element " $i$ " is:

$$
E_{i}=\frac{m_{i} v_{i}^{2}}{2}+\frac{J_{i} \omega_{i}^{2}}{2}
$$

where: $\frac{m_{i} v_{i}^{2}}{2}$ is the kinetic energy of the translation with the speed $v_{i}$;

$$
\begin{aligned}
& \frac{J_{i} \omega_{i}^{2}}{2} \text { - kinetic energy of the rotation of the element; } \\
& m_{i} \text { - mass of the element; } \\
& \quad J_{i} \text { - inertia moment of the element. }
\end{aligned}
$$

Considering the reference point " $k$ " with the known speed $v_{k}$, where is concentrated the reduced mass $M_{r e d}$ in concordance with the law of the conservation of kinetic energy, the following equation can be written:

$$
\frac{M_{r e d} v_{k}^{2}}{2}=\sum_{i=1}^{n}\left(\frac{m_{i} v_{i}^{2}}{2}+\frac{J_{i} \omega_{i}^{2}}{2}\right)
$$

hence, it results:

$$
M_{\text {red }}=\sum_{i=1}^{n}\left[m_{i}\left(\frac{v_{i}}{v_{k}}\right)^{2}+J_{i}\left(\frac{\omega_{i}}{\omega_{k}}\right)^{2}\right] .
$$

Similarly, the next expression for the reference point " $k$ " with rotational motion, can be obtained:

$$
\frac{J_{r e d} \omega_{k}^{2}}{2}=\sum_{i=1}^{n}\left(\frac{m_{i} v_{i}^{2}}{2}+J_{i} \frac{\omega_{i}^{2}}{2}\right)
$$

hence, the reduced moment becomes:

$$
J_{\text {red }}=\sum_{i=1}^{n}\left[m_{i}\left(\frac{v_{i}}{\omega_{k}}\right)^{2}+J_{i}\left(\frac{\omega_{i}}{\omega_{k}}\right)^{2}\right] .
$$

The reduced moment of the active forces can be defined considering the balance between its elementary mechanical work and elementary mechanical work of the component parts of the drive mechanism,

$$
F_{r e d} d x_{k} \cos \alpha_{k}=\sum_{1}^{n}\left(F_{i} d x_{i} \cos \alpha_{i}+M_{i} d \alpha_{i}\right)
$$

If the above expression is derived with respect to time, it becomes:

$$
F_{\text {red }} v_{k} \cos \alpha_{k}=\sum_{1}^{n}\left(F_{i} v_{i} \cos \alpha_{i}+M_{i} \omega_{i}\right)
$$


or:

$$
F_{r e d}=\frac{1}{\cos \alpha} \sum_{1}^{n}\left(F_{i} \frac{v_{i}}{v_{k}} \cos \alpha_{i}+M_{i} \frac{\omega_{i}}{\omega_{k}}\right),
$$

where:

$F, M_{i}$ are the force and the moment applied to the element " $i$ "; $v_{i}$ - the speed of the application point of the force $F_{i}$;

$\alpha_{i}$ - the angle between the vectors $\overline{v_{i}}$ and $\overline{F_{i}}$;

$v_{k}$ - the speed of the application point of the force $F_{k}$;

$\alpha_{k}$ - the angle between the vectors $\overline{v_{k}}$ and $\overline{F_{k}}$.

In order to establish the reduced moment, the method is the same as previously. Hence, the expression of the reduced moment is:

$$
M_{r e d} \omega_{k}=\sum_{1}^{n}\left(F_{i} \cos \alpha_{i}+M_{i} \omega_{i}\right)
$$

or,

$$
M_{r e d}=\sum_{1}^{n}\left(F_{i} \frac{v_{i}}{\omega_{k}} \cos \alpha_{i}+M_{i} \frac{\omega_{i}}{\omega_{k}}\right)
$$

The analytical study of the behaviour of the power contactors drive mechanism during dynamic switching conditions, requires a mathematical model and also an experimental model in order to validate the proposed mathematical ones.

The strength characteristic of the drive mechanism, in the case of a power contactor means the variation of the resultant strength force $F_{R}$ vs. the air gap $\delta$. This characteristic has discontinuities because of the prestressed springs of the main contacts and auxiliary contacts, Figure 1.

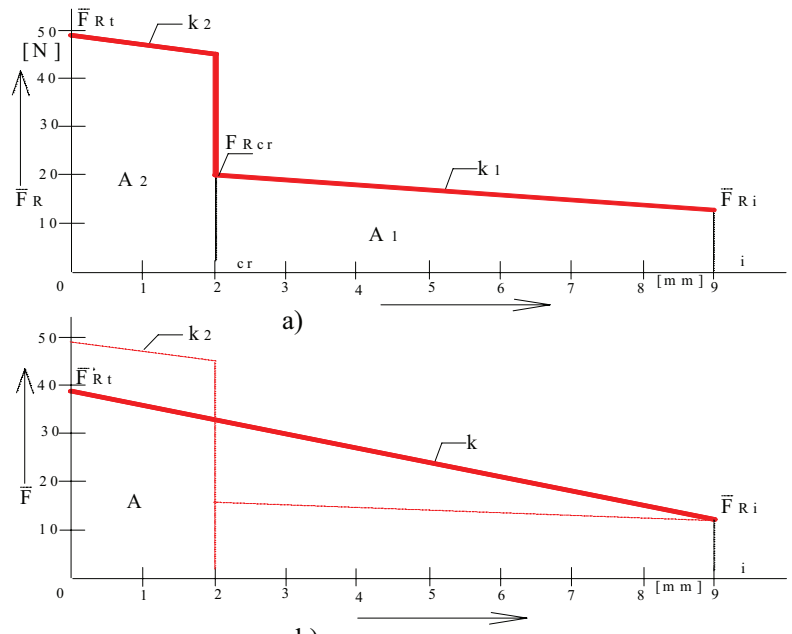

b)

Figure 1. The strength characteristic of the contactor: a) with one single jump; b) without any jump.
This type of characteristic can be idealized with a single jump, Figure $1 \mathrm{a}$, at critical air gap, $\delta_{c r}$, when the prestressed springs of the main contacts start to act. The trapeze areas $A_{1}$ and $A_{2}\left(A_{1}=\frac{F_{R c r}+F_{R i}}{2}\left(\delta_{i}-\delta_{c r}\right) ; A_{2}=\frac{F_{R c r}+F_{R t}}{2} \delta_{c r}\right)$, are direct proportional with the required energy of the open spring and other prestressed springs. Also, the characteristic can be idealized with a single line, without any jump, Figure $1 b$, where the effect of all springs can be considered equal with an equivalent spring. The area $A$ is equal with the sum of the above areas, $A=A_{1}+A_{2}, A=\frac{F_{R c r}+F_{R i}}{2} \delta_{i}$. This characteristic can be used with the aim to optimize the response time of the drive mechanism.

The mathematical model of the drive mechanism allow to make analysis about the behaviour of the mechanical system of the power contactors during transient conditions of the dynamic switching, as connection and disconnection. A solution may consider the idealized strength characteristic as presented in Figure 1a, which includes two equivalent springs with the elastic constants, $k_{1}$ and $k_{2}$. At the first spring, the mechanical tension begins at the air gap value of $\delta=\delta_{i}=\delta_{0}$, and the second spring, the mechanical tension begins from the critical air gap $\delta=\delta_{c r}$, which because of the pretension energy there is the jump from the characteristic. Other solution, may takes into consideration only one equivalent spring, as presented in Figure 1b.

In order to analyse the behaviour of the drive mechanism of the power contactor during transient conditions (connection/disconnection), it has to investigate the following aspects:

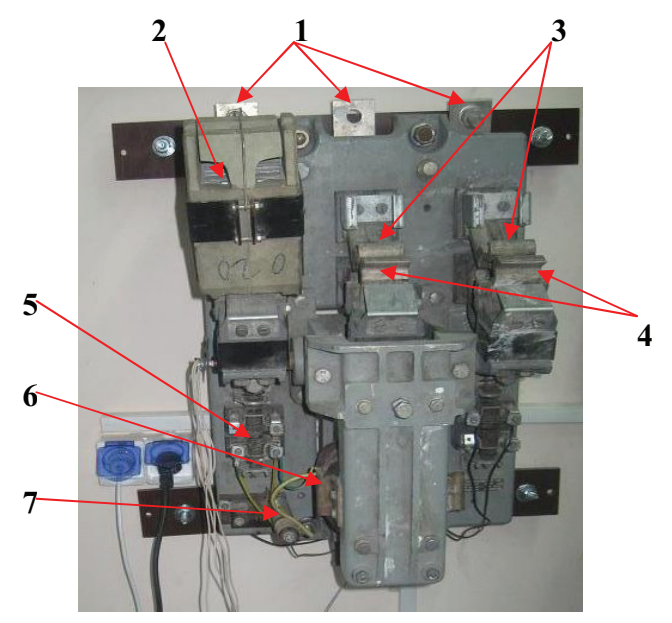

Figure 2. Main component parts of the AC three-phase contactor. 
- time evolution of the trip of the movable armature;

- time evolution of the force/torque of the movable armature;

- connection time and disconnection time.

\section{Experimental Tests}

The analyzed power AC three-phase contactor has the following rated data: rated voltage of $500 \mathrm{~V}$, rated current of $600 \mathrm{~A}$ and the rated frequency of $50 \mathrm{~Hz}$. The drive coil has the rated voltage of $220 \mathrm{~V}$ and the rated frequency of $50 \mathrm{~Hz}$. The main component parts of the power contactor, as presented in Figure 2, are: 1 - three-phase terminals, 2 - extinguish chamber for the electric arc, 3 - fixed power contacts, 4 - movable power contacts, 5 - auxiliary contacts, 6 - drive coil, 7 - limiting resistor.

This type of power contactor has the movable armature with the rotational motion. Hence, the strength characteristic will be the variation of the strength torque vs. angle of the rotation of the shaft where is placed the movable armature of the power contactor. Using a digital dynamometer,

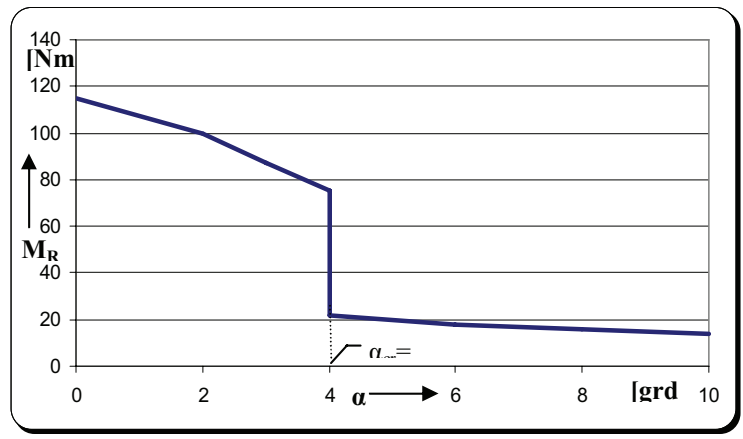

Figure 3. The strength characteristic of the analyzed contactor.

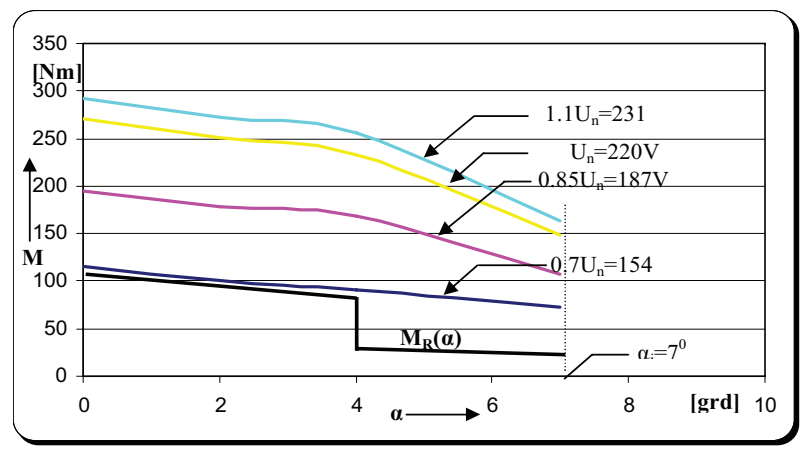

Figure 4. The comparison between the strength characteristic and the mechanical characteristics of the drive coil at different voltage supply. the strength characteristic has been obtained, Figure 3. It can be observed that the contribution to the total strength torque of the auxiliary contacts on this characteristic is negligible.

The comparison between strength torque characteristic and the mechanical characteristics of the drive coil at different voltage supply, is shown in Figure 4. It can be noticed a good correlation between strength and mechanical characteristics and the power contactor can work till the minimum voltage supply of $154 \mathrm{~V}\left(0.7 \mathrm{U}_{\mathrm{n}}\right)$ for the drive coil. At this voltage supply, the strength characteristic is still under the mechanical characteristic of the drive coil.

In order to get the time evolution characteristics of the trip of the movable armature, an experimental set-up has been achieved, as presented in Figure 5.

A potentiometer of $1 \mathrm{k} \Omega$ value has been mounted with the adjustable part in the shaft of the power contactor where are mounted the movable armature and the power movable contacts. This potentiometer is supplied with $\mathrm{dc}$ voltage about $30 \mathrm{Vdc}$, using a single-phase transformer $\mathrm{Tr}$, a rectifier bridge and a filter capacitor of $470 \mathrm{uF} / 40 \mathrm{~V}$ with the aim to obtain a smooth rectified dc voltage. When the shaft begins to rotate, in the same time, the adjustable part

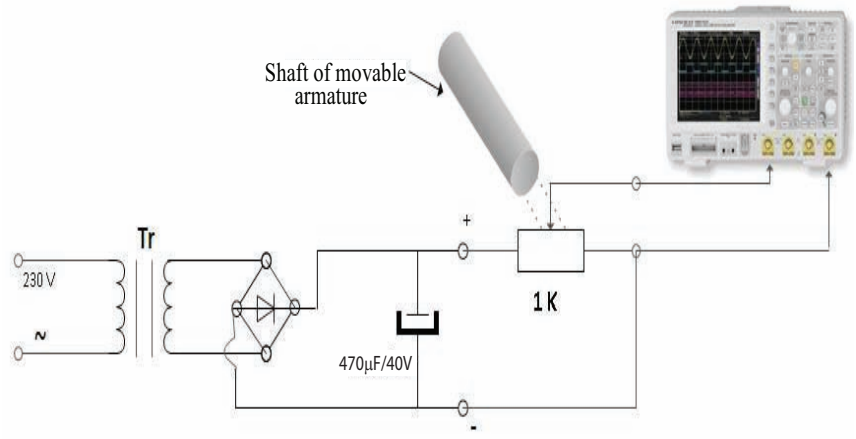

Figure 5. Experimental set-up.

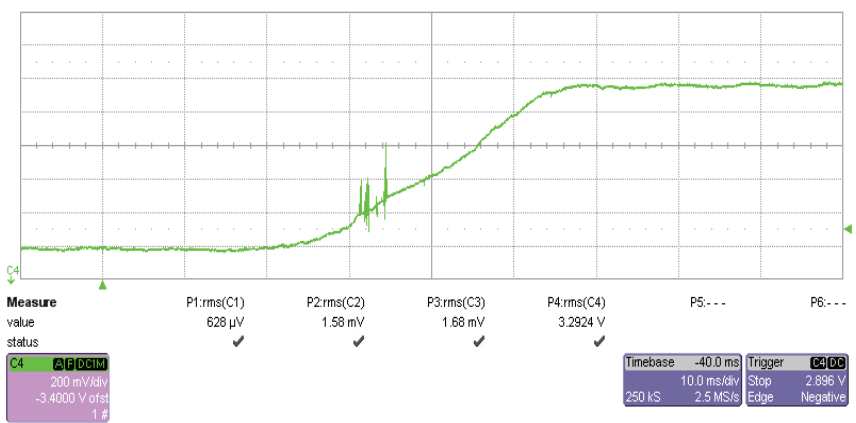

Figure 6. Trip characteristic of the contactor during connection when the voltage supply of the drive coil is $220 \mathrm{~V}$. 


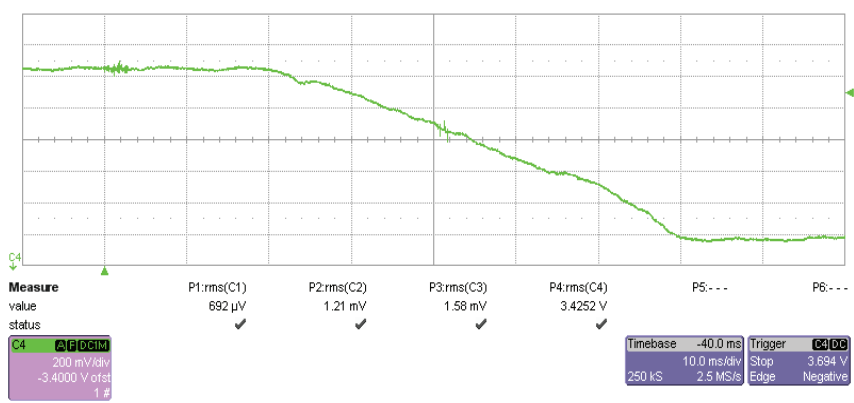

Figure 7. Trip characteristic of the contactor during disconnection when the voltage supply of the drive coil is $220 \mathrm{~V}$.

Table 1. Connection and disconnection time at different voltage supply of the drive coil

\begin{tabular}{ccc}
\hline $\mathrm{U}_{\text {coil }}[\mathrm{V}]$ & $\mathrm{t}_{\text {con }}[\mathrm{ms}]$ & $\mathrm{t}_{\text {discon }}[\mathrm{ms}]$ \\
\hline 242 & 22 & 50 \\
231 & 24 & 50 \\
220 & 30 & 50 \\
187 & 35 & 48 \\
154 & 50 & 45 \\
125 & 100 & 67 \\
\hline
\end{tabular}

of the potentiometer starts to rotate, and the resistance of the potentiometer will vary direct proportional with the main shaft rotation. It results that the voltage variation across the potentiometer is direct proportional with the rotation of the shaft of the power contactor. This voltage is recorded using a digital oscilloscope, Figure 5. The recorded oscillograms for both connection and disconnection cases of the power contactor at different voltage supply of the drive coil, are presented below, from Figure 6 to Figure 9.

The first two oscillograms, Figure 6 and Figure 7, means the time evolution of the trip in the case of connection and disconnection of the power contactor, when the voltage supply of the drive coil was the rated value of $220 \mathrm{~V}$. It can be noticed the connection time of $30 \mathrm{~ms}$ and the disconnection time of $50 \mathrm{~ms}$.

The last two oscillograms, Figure 8 and Figure 9, show the same time evolutions of the trip but in the situation when the drive coil of the power contactor has been supplied with the minimum voltage of $154 \mathrm{~V}\left(0.7 \mathrm{U}_{\mathrm{n}}\right)$. It can be noticed a value of $45 \mathrm{~ms}$ for the connection time, so an

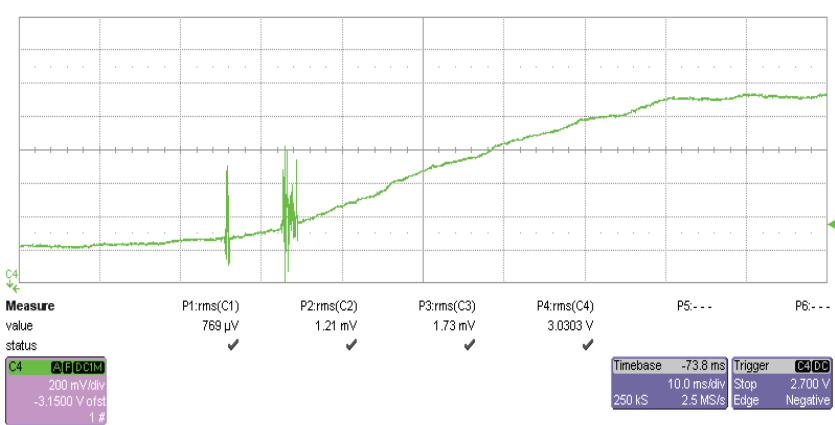

Figure 8. Trip characteristic of the contactor during connection when the voltage supply of the drive coil is $154 \mathrm{~V}$.

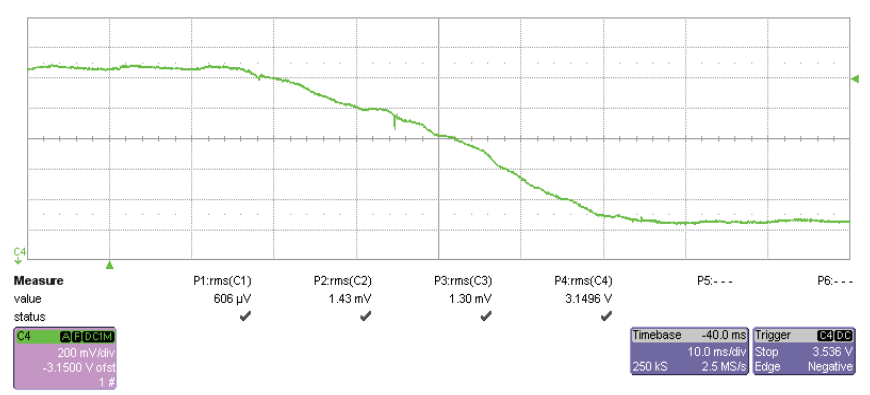

Figure 9. Trip characteristic of the contactor during disconnection when the voltage supply of the drive coil is $154 \mathrm{~V}$.

increased value respect to the case when the drive voltage has been supplied with the rated value of $220 \mathrm{~V}$. The other values for the connection and disconnection time, are synthesized in the Table 1 below.

It can be observed an increasing of the connection time when the voltage supply of the drive coil is decreased, because the trip force of the coil depends proportional on this voltage supply, and the higher the value of the trip force, the less the value of the connection time.

\section{Conclusions}

Contactors with AC coils are among the most widely utilized electromechanical devices in industry, especially for motor starting operation. One of the important key-feature for contactors, is represented by its drive mechanism. The behaviour of its drive mechanism during transient conditions, such as connection or disconnection of the main electric circuit, affects the electric drive system from an entire automation process. Hence, it is of major importance to study the drive mechanism of the contactors, 
especially the correlation between strength characteristic and electromagnetic drive characteristics. Another important parameters which depend on the drive mechanism, are the connection and disconnection times during switching operations of the contactor. At a certain voltage supply of the drive coil, an optimum connection time has to be established.

\section{Acknowledgements}

This work was supported by CNCSIS - UEFISCDI, project number 610 PNII - CAPACITATI, 2013.

\section{References}

1. Collins E R, and Bridgwood M A (1997). The impact of power system disturbances on AC-coil contactors, IEEE 1997 Annual Textile, Fiber, and Film Industry Technical Conference.

2. Shareef H, Marzuki N et al. (2010). Experimental investigation of ac contactor ride through capability during voltage sag, 9th International Conference on Environment and Electrical Engineering, 325-328.

3. Hardi S, and Daut I (2010). Sensitivity of low voltage consumer equipment to voltage sags, 4th International Power Engineering and Optimization Conference, 396-401.

4. Silva S M, Braga M F et al. (2007). Analysis and development of a ride-through device for ac contactors, European Conference on Power Electronics and Applications, 1-9.

5. Pires I A, Filho B J C et al. (2012). Protecting control panels against voltage sags using square-wave series voltage compensators, IEEE Industry Applications Society Annual Meeting, 1-7.

6. Akolkar S M, and Kushare B E (2010). Effect of point on wave angle on sensitivity of AC coil contactor and SMPS to voltage sags, Conference Proceedings IPEC, 957-961.

7. Leung C, Streicher E et al. (2003). Microstructure effect on reignition and welding properties of copper-tungsten electric contact, Proceedings of the Forty-Ninth IEEE Holm Conference on Electrical Contacts, 132-138.

8. Krätzschmar A, Herbst R et al. (2010). Basic investigations on the behavior of advanced ag/sno2 materials for contactor applications, Proceedings of the 56th IEEE Holm Conference on Electrical Contacts, 1-7.

9. Shea J J (2008). High current ac break arc contact erosion, Proceedings of the 54th IEEE Holm Conference on Electrical Contacts, xxii-xlvi.
10. Nitu S, Pavelescu D et al. (2009). Ecological solutions for power switching apparatus, IEEE EUROCON, 637-642.

11. Nițucă C (2013). Thermal analysis of electrical contacts from pantograph-catenary system for power supply of electric vehicles, Electric Power Systems Research, vol 96, 211-217.

12. Ruiz R, Espinosa J-R et al. (2010). A computer model for teaching the dynamic behavior of ac contactors, IEEE Transactions on Education, vol 53(2), 248-256.

13. Wada M, Yoshimoto $H$ et al. (2002). Dynamic analysis and simulation of electromagnetic contactors with AC solenoids, IEEE 28th Annual Conference of the Industrial Electronics Society, vol 4, 2745-2751.

14. Albu M M, Holbert K E et al. (2004). Embedding remote experimentation in power engineering education, IEEE Transactions on Power Systems, vol 19(1), 139-143.

15. Chiriac G (2012). Thermal analysis of fuses with variable cross-section fuselinks, Electric Power Systems Research, vol $92,73-80$.

16. Jinbo Z, Shushu L et al. (2010). Research and realization of online and non-contact infrared temperature measurement of high-voltage switch contactors, International Conference on Measuring Technology and Mechatronics Automation, 1047-1050.

17. Cairoli P, Dougal R A et al. (2013). Coordination between supply power converters and contactors for fault protection in multi-terminal MVDC distribution systems, IEEE Electric Ship Technologies Symposium, 493-499.

18. Cantemir L, Nituca C et al. (2011). Unconventional current collection from a contact line for electric traction vehicles, International Conference on Pantograph Catenary Interaction Framework for Intelligent Control, Amiens, France.

19. Tiley G L (1987). Effect of mine hoist drives on the electrical power supply system, IEEE Transactions on Industry Applications, IA-23, 742-747.

20. Duguay L (1996). System design concepts for a compact power system featuring $250 \mathrm{~W}$ rectifiers, 18th International Telecommunications Energy Conference, 220-226.

21. Nituca C (2013). Thermal analysis for a double sided linear induction motor, European Scientific Journal, vol 9(9), $38-50$

22. Swamy M M, Kume T et al. (2006). Extended high-speed operation via electronic winding-change method for $\mathrm{AC}$ motors, IEEE Transactions on Industry Applications, vol 42(3), 742-752.

23. Ghazala A A (2009). Retrofit implementation of medium voltage contactors at Abou Quir power station in Egypt, IEEE PowerTech, Bucharest, 1-6. 